\title{
Collection, transfer and processing of information in systems of monitoring of objects based on wireless sensor networks
}

\author{
Maxim Sergievskiy ${ }^{1,2, a}$ and Sergey Syroezhkin ${ }^{1}$ \\ ${ }_{1}^{1}$ National Research Nuclear University MEPhl, 115409 Moscow, Russia \\ ${ }^{2}$ Moscow Technological Institute, 119334 Moscow, Russia
}

\begin{abstract}
Monitoring of the aircraft structures' during the pre-flight testing is a critical task of the aerospace industry. One of the most promising solutions, not yet widely applied, is continuous monitoring of aircraft structures using wireless sensor network technology. The brief summary of the proposed system is the following: special sensors send signals to the local motes (autonomous computing device equipped with a wireless transmitter). Information from motes is gathered by routers which then transfer the aggregated information to the datacenter. Applications of corporate network control and define flexible patterns for processing of the information received from sensors. This network structure allows to centralize data collection modes in the process of testing; implement continuous data collection at a defined frequency; process and display data in real-time.
\end{abstract}

\section{Introduction}

Internet of Things (IoT) - is an environment, which provides an interface for a variety of objects, equipped with computing and communication resources. In this case, Internet is understood broadly, not only as a method of transmitting data within a network using specific protocols and technologies, but also as a set of different services. Obviously, such a definition of IoT is very close to the definition of wireless sensor networks (WSN) [1]. However, the concept of IoT is broader than WSN concept, because the IoT brings together, firstly, animate and inanimate objects, and, secondly, the objects that do not have an active communication tools [2].

WSN consists of a number of small computational and communication devices - motes. Mote is a board, which size is usually less than one cubic inch, which consists of: a processor, memory (Flash and RAM), digital-to-analog and analog-to-digital converters, RF transceiver, power supply and sensors.

The sensors are connected to the board via the digital and analog connectors and can be very diverse: temperature, pressure, humidity, light, vibration, electromagnetic, chemical and others. The set of installed on mote sensors depends on the functions performed by the WSN. As the power source nodes use small batteries; typically, rechargeable batteries are not applied, since its charging is impossible in most cases. Motes serve for the collection, basic processing and transmission of the data.

Gateway is a special network's node, a sufficiently powerful computer, which performs the main functional processing of collected data. To retrieve data from the motes, node is equipped with a radio transmitter. Because of the limited range of the transmitter, gateway does not receive information directly from every network's mote, it can obtain data just from the motes that are located close to it. The problem of obtaining sensors data by gateway is solved as follows: motes can communicate with each other using transceivers operating in the radio bands. They send, information collected by motes sensors, as well as service information (status of devices and the results of data transmission).

Information is transferred from one mote to another by chain, and finally nearest to gateway motes send all the collected data to it. If some motes fail, the sensor network operation should continue after reconfiguration and changing its structure. But in this case, of course, the number of motes, sources of information, decreases.

To perform the functions a special operating system is installed on each node. Currently one of the most common operating system for WSN is TinyOS [3]. It is event-driven real-time operating system, designed to work in limited computing resources environment. This OS, as well as a number of others $[4,5]$, has special libraries and protocols that allow motes automatically establish communications with neighboring items to form a sensor network of a given topology. The controlling program of wireless sensor node describes all its operation logic, including such basic things as protocols of interaction with hardware, the primary processing of sensory data, and more complex processes, such as routing algorithms, encryption and data transmission.

The most important factor when working with wireless sensor networks is a limited capacity of the battery, mounted on mote [1]. It should be taken into account, that it is impossible to replace the battery in

\footnotetext{
a Corresponding author: sermax@yandex.ru
} 
many cases. For this reason, firstly, motes should perform only simple primary processing, aimed at reducing the amount of transmitted information, and secondly, perhaps the most important, the number of data transmission and reception cycles should be minimized. To solve this issue special communication protocols were developed. The most common of which are the protocols of the ZigBee Alliance [6], which were created to coordinate activities of wireless sensor technologies. In general, to produce a standard, including protocol stack for wireless sensor networks, ZigBee used the previously developed the IEEE 802.15.4 standard, which specifies physical and medium access layers for wireless data transmission over short distances (up to $75 \mathrm{~m}$ ) with low power consumption, but high degree of reliability.

The main purpose of the use of sensor networks is to control the state of objects during its operation. The critical components and systems can be equipped with a wireless data collection systems and diagnostic tools, for analysis and decision-making [7].

\section{Problem statement}

Now let's discuss a solution of important task - the control of modern aircraft structures' state in the process of testing and operation. This problem is relevant for the aircraft industry, because hidden defects caused by mechanical damage can lead to emergencies and accidents. Different manufacturers solve the task of improving the reliability and safety of the aircraft differently. They perform more preflight tests, analyze results and make estimates.

One of the most promising ways to solve this problem, not widespread yet, is the continuous monitoring of aircraft constructions in the process of testing and operation with the output on the dashboard of the necessary information. There are all preconditions to populate this approach:

- wide range of compact sensors (strain, temperature, pressure, vibration, location, etc.) with different mounting options;

- wireless sensor technology to collect information from sensors and send it to consumers; wide capabilities of control of information gathering modes;

- data processing techniques, implemented by custom applications.

The main aim of this work is to create a wireless system for monitoring of aircraft structures' deformation level during static and dynamic tests.

\section{System architecture}

The structure of the monitoring systems in most cases is the following: there are sensors connected to motes on the lower level. Several sensors can be connected to one mote; it is possible that the sensors are located on the mote's board - it depends on the tasks, which sensors perform. In our case, sensors are mounted directly on a controlled structure.
Motes are only used for collecting and converting the primary sensor data, which they send to each other and, eventually, to a special unit - gateway (base station) that has a wire connection to the server (the network). The network may contain multiple gateways; it increases its reliability and performance. Controlling of information collection modes and main processing of the obtained data is performed by applications on the corporate network. This approach allows to get rid of a large number of cable connections, since before wire connections were widely used in the airspace industry [8].

In case, when sensors are very tight, a star topology is considered to as the most effective. In this case, motes will transmit information to special routers that communicate with the base station. Routers are not equipped with sensors.

Thus, the network consists of a set of clusters, each formed by a router and motes with sensors, polled by router. Routers forward data to each other and, finally, the data comes to the gateway. The reliability of such systems is very high: even failure of a large number of motes or routers will not affect the operation of the system. Additionally, routers may be located outside of the deformable structure [9]. Fig. 1 shows a monitoring system consisting of a gateway router and sensor motes. Each mote has several sensors, connected to it by wires. To save energy motes are unable to receive and retransmit data from other motes, they only can send to router their own data.

Some routers have a direct link with the gateway, while others are transferring data by a longer chain, using other routers. This approach has both advantages and disadvantages. On one hand, it allows the use of only one gateway, but on the other, it increases utilization of some of the routers. Since in this domain network topology usually varies little during operation, these routers will fail earlier than others. Knowing the characteristics of a particular network topology makes it possible to increase the number of routers and efficiently balance the load between them, extending the network lifetime and avoiding potential loss of data.

The described network architecture can be attributed to the hybrid type, where some nodes (routers) form a mesh network, and the router and its motes actually use the cluster topology. An important task that WSN developer is faced is to increase the operating time of the network without having to replace the batteries. The most energy-consuming operations for wireless node is sending and receiving data. In the process of deformation level monitoring of aircraft constructions during static and dynamic tests measuring frequency reaches $200 \mathrm{~Hz}$, that is, WSN should transmit a quite large data stream $[10,11,12]$.

Let's determine the amount of payload data that should be transmitted to the gateway over the network, consisting of $\mathrm{N}$ motes with $\mathrm{M}$ sensors on each, if one deformation level measurement takes two bytes of memory.

$$
\mathrm{Q}=2 \times \mathrm{N} \times \mathrm{M}(\mathrm{B})
$$




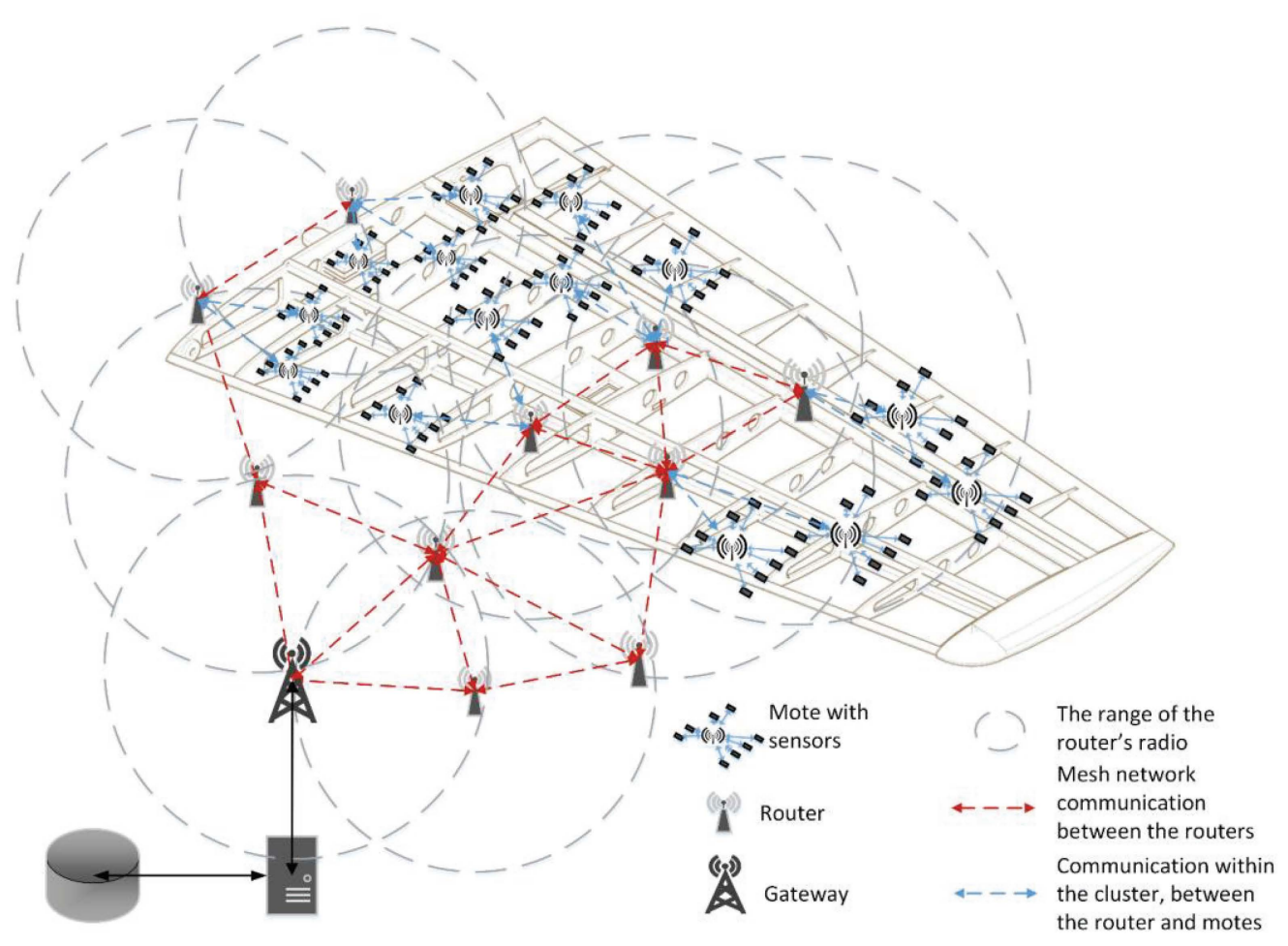

Figure 1. Structure of monitoring system

If the required measurement frequency of deformation is $\mathrm{H} \mathrm{Hz}$, the flow of information transmitted over the network as a whole, will be equal to:

$$
\mathrm{S}=\mathrm{Q} \times \mathrm{H}=2 \mathrm{~N} \times \mathrm{M} \times \mathrm{H}(\mathrm{Bps}) .
$$

For example, for a network consisting of 100 motes with 10 sensors on each, and a measurement frequency of $10 \mathrm{~Hz}$, the flow of information will be $20 \mathrm{~KB} / \mathrm{s}$. This estimate does not take into account the fact that the overhead information, accompanying each message transmitted over the network, increases the total, transmitted over the network, traffic in about 7-10 times. Thus, the actual flow in this case is equal to 140$200 \mathrm{~KB} / \mathrm{s}$. Note, this is an estimate of the total data stream that arrives at the gateway, for routers and, especially, motes values of local flows will be significantly lower. Continuous processing and transmission of the data stream may trigger certain network components (routers or motes) failure in some cases. In order to maximize the network lifetime, you can apply several solutions related to the choice of its functioning modes. Let's discuss them in the next paragraph.

The process of developing of wireless deformation level monitoring system includes not only determining the network structure and configuration, but the solution of two interconnected problems - choice of hardware components and software creation.

\section{Hardware components}

To measure the deformation level of aircraft structures we use strain gauges model "FLA-5-17" by Tokyo Sokki
Kenkyujo Company. Basic characteristics of the sensors are shown in the Table 1.

Table 1. Basic characteristics of the sensors.

\begin{tabular}{|c|c|c|c|}
\hline $\begin{array}{c}\text { Applica- } \\
\text { ble } \\
\text { specimen }\end{array}$ & $\begin{array}{c}\text { Metal, } \\
\text { Glass, } \\
\text { Ceramics }\end{array}$ & Backing & Epoxy \\
\hline $\begin{array}{c}\text { Operati- } \\
\text { onal temp. } \\
\left({ }^{\circ} \mathbf{C}\right)\end{array}$ & $\begin{array}{c}-20 \\
\sim+80^{\circ} \mathrm{C}\end{array}$ & Element & $\mathrm{Cu}-\mathrm{Ni}$ \\
\hline $\begin{array}{c}\text { Temp. } \\
\text { compensa- } \\
\text { tion } \\
\left.\text { range( }{ }^{\circ} \mathrm{C}\right)\end{array}$ & $\begin{array}{c}+10 \sim+80 \\
{ }^{\circ} \mathrm{C}\end{array}$ & Strain limit & $\begin{array}{c}5 \% \\
\left(50000 \times 10^{-}\right. \\
\left.{ }^{\text {strain }}\right)\end{array}$ \\
\hline $\begin{array}{c}\text { Bonding } \\
\text { adhesive }\end{array}$ & $\begin{array}{c}\mathrm{CN}, \mathrm{P}-2, \\
\mathrm{~EB}-2\end{array}$ & $\begin{array}{c}\text { Fatigue life } \\
\text { at room } \\
\text { temp. }\end{array}$ & $\begin{array}{c}1 \times 10^{6} \\
\left( \pm 1500 \times 10^{-}\right. \\
\left.{ }^{6} \text { strain }\right)\end{array}$ \\
\hline
\end{tabular}

As a hardware platform for primary data processing and transmission, we use motes IRIS (Fig. 2) from MEMSIC.

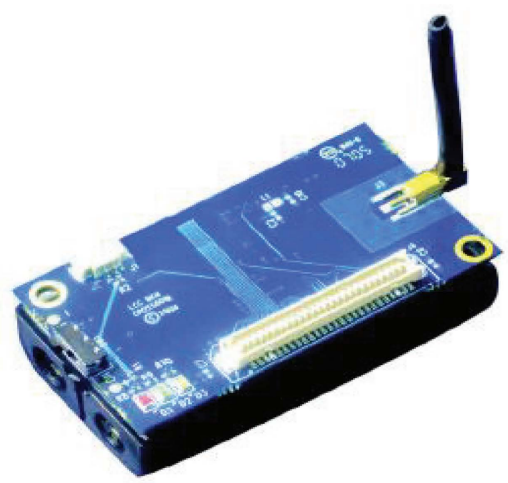

Figure 2. Mote IRIS 
They have a wide range of features including built-in processor, ADC, multi-channel radio transmitter, RAM, and interface for connecting sensors [3].

Special data captures and interface board are used in this WSN for strain monitoring. Both of these cards connect to themote through the 51-pin interface. To connect strain gauges FLA-5-17 to wireless motes IRIS a special data capturing board MDA300CA is used. This Board is attached to the IRIS Mote's extension connector and provides a set of digital and analog inputs for connecting a variety of sensors.

Every strain gauge connects to a data capturing board through any differential pair of channels and $\mathrm{Ai}+\mathrm{Ai}-$, which are used for connecting external sensors with a gain of 100 . Thus, a single board can connect up to 10 sensors of the strain level. The dynamic range of channels is $\pm 12.5 \mathrm{mV}$. The result obtained after analog-to-digital conversion of the sensor readings, can be translated into a voltage $\mathrm{V}(\mathrm{mV})$ according to the following formula:

$$
\mathrm{V}=12,5 \times(\mathrm{ADC} / 2048-1)
$$

For routers, we use the same IRIS motes, but they are not equipped with sensors. Sensor readings received by mote, should be transferred wirelessly to the server for further processing.

Special multi-purpose USB-interface board MIB520 (Fig. 3) of MEMSIC is the link between the wireless sensor network and the server. This board (gateway) with a transmitter module, integrated with IRIS mote, on one hand is able to exchange messages with the wireless network nodes, and on the other to transmit and receive data via USB-port of an ordinary computer.

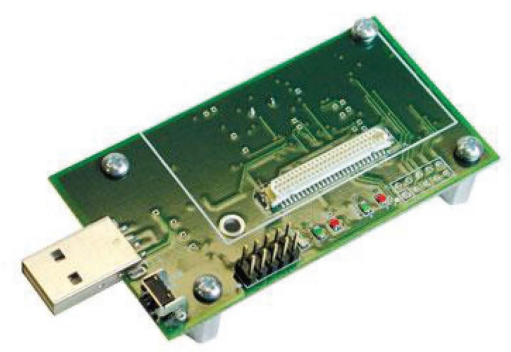

Figure 3. MIB520 board

\section{Software components}

For development of software components of condition monitoring system, we use a special platform MoteWorks of Crossbow Company [3]. This platform is based on the TinyOS operating system. Developers of the MoteWorks platform considered features of such systems and created the product, which allows create effective and reliable decisions.

The monitoring system software has two groups of components: network and server. Network components include a control application for motes and special software, to ensure the normal functioning of the network. Control application implements all algorithm required for monitoring and data transmission. For the data transfer, application uses stack of network protocols XMesh of MoteWorks platform. The stack of protocols XMesh supports multilink transmission of messages and conforms to the standard 802.15.4 [13].

Server software components of the monitoring system are running on the server special services, with main tasks to 1) collect, 2) store and 3) process the readings obtained by the network sensor. Server components uses XServe platform of MoteWorks as a basis, which collects incoming data on the interface card, converts them to a format specified by XML- template, and stores in a special database.

While monitoring of the aircraft structures state, experts rarely analyze obtained sensor data in the realtime, often they study them after experiments. Thus, often there is no need to deliver sensors data on gateway in real-time, and hence, a better approach from the standpoint of energy saving, would be an accumulation of a certain amount of data at the node, its compression, and only then transmission to the network. This operating mode allows you to switch the radio transmitter in the sleep mode, and thus save energy. In addition, it will allow solve another problem - avoiding conflicts arising from the simultaneous transmission of data from different routers.

Dependency of energy consumption on data transmission distance is elevated, which means that in

addition to the transmission time, the distance between transmitter and receiver and the transmitter's power also determines energy consumption. To reduce the cost of energy, required to data transmission, it is important to design the network in such a way to prevent the transmission over a long distances. Furthermore, the configuration of the domain allows us to pre-arrange the location of nodes, and hence it becomes possible to calculate the transmitter power that is required for reliable operation of the network.

In practice it is desirable to use minimum transmitter power, as this will not only reduce the energy consumption for data transmission, but also reduce the number of possible collisions [14].

\section{Conclusions}

In conclusion we highlight the key advantages of the use of wireless sensor networks for controlling and monitoring aircraft structures:

- lack of cables or a minimum number of cables;

- very small form factor of motes that basically allows to embed them in the body of various aircraft structures or connect to various devices;

- reliability of individual elements, and the whole system in the process of information collection and transmission; - minimum energy consumption for receiving and transmitting radio signals, which allows you to use compact batteries;

- simplicity of a sensor network self-organization;

- scalability, allowing to ensure the deployment of networks with large number of nodes, which is important for a detailed analysis of aircraft structures. 
The use of aircraft structures monitoring system, created on the base of wireless sensor network technology, will allow to organize the centralized management of the data collection modes during experiments; to carry out continuous data collection from sensors of various type with the set frequency of measurements; quickly to process and display data in real time.

\section{References}

1. C. Cordeiro and D. Agrawal, Ad hoc and sensor networks: theory and applications (World Scientific Pub. Co., 2006)

2. J. Hart and K. Martinez, Earth-Science Reviews, 78, 177 (2006)

3. Crossbow Technology, (2014) www.xbow.com

4. UC Berkeley. TinyOS Open WSN project (2013). http://www.tinyos.net

5. A.S. Kosachev and V.N. Ponomarenko, Real time operating systems (Institute of System Programming, 2006)

6. C. Shen, S. Harte, E. Popovici, B. O'Flynn and R. Atk, Electronics Letters, 45, 1098 (2009)

7. A. Mainwaring, J. Polastre, R. Szewcyk and D. Culler, ACM International Workshop on Wireless Sensor Networks and Applications (2002)

8. T. Haenselmann, GFDL Wireless Sensor Network textbook, (2006) http://pi4.informatik.uni-mannheim. $\mathrm{de} / \sim$ haensel/sn_book

9. A.J. Swati, R. Priyanka, International Journal on Computer Science and Engineering, 2, 3089 (2010)

10. R. Kay and F. Mattern, IEEE Wireless Communications, 11, 54 (2011)

11. E.F. Nakamura, A.A.F. Loureiro and A.C. Frery, ACM Computing Surveys, 39, 9 (2007)

12. M.V. Sergievskiy and S. N. Syroezhkin, Modern Applied Science; 9, (2015)

13. Linear Technology (2013). www.dustnetworks.com

14. A. Tiwari, P. Ballal and F.L. Lewis, ACM Transactions on Sensor Networks, 3, 1 (2007) 